\title{
TISSUE DOPPLER IMAGING IN VERY PRETERM INFANTS. RELATION TO BLOOD PRESSURE AND SUPERIOR VENA CAVA FLOW
}

\author{
A. Lee ${ }^{1,2}$, E. Nestaas ${ }^{3}$, K. Liestøl ${ }^{4}$, L. Brunvand ${ }^{5}$, R. Lindemann ${ }^{2,6}$, D. Fugelseth ${ }^{2,6}$
}

${ }^{I}$ Childrens Clinic, Akershus University Hospital, Lørenskog, ${ }^{2}$ Department of Neonatal Intensive Care, Oslo University Hospital, Ullevål, Oslo, ${ }^{3}$ Department of Paediatrics, Vestfold Hospital Trust, Tønsberg, ${ }^{4}$ Department of Informatics, University of Oslo, ${ }^{5}$ Department of Paediatrics, Oslo University Hospital, Rikshospitalet, ${ }^{6}$ Faculty of Medicine, University of Oslo, Oslo, Norway

Background and aims: Cardiovascular monitoring of the preterm is challenging. Blood pressure (BP) is an indirect measure of cardiovascular function.

Superior vena cava (SVC) flow has become a surrogate measure of systemic flow. Tissue Doppler imaging (TDI) is a new tool for measuring myocardial function.

The aim of this study was to explore the relation of SVC flow and BP to myocardial function measured by TDI in newborn, preterm infants.

Methods: Fourty-eight infants (median (range) gestational age 27.5 (24-32) weeks, birth weight 1069 (495$1620) \mathrm{g}$ ) underwent echocardiographic examination 5 (2-8) hours after birth. Annular velocities and displacement were measured with TDI, and SVC flow by conventional echocardiography with simultaneous BP measurements.

Results:

\begin{tabular}{|c|c|c|}
\hline & Mean (SD) & $\mathbf{n}$ \\
\hline SVC flow (ml/kg/min) & $89(44)$ & 47 \\
\hline Mean arterial BP $(\mathrm{mmHg})$ & $33(7)$ & 45 \\
\hline Systolic BP (mmHg) & $38(16)$ & 45 \\
\hline Diastolic BP (mmHg) & $24(11)$ & 45 \\
\hline $\begin{array}{l}\text { Peak systolic annular velocity LV } \\
(\mathrm{cm} / \mathrm{s})\end{array}$ & $2.0(0.7)$ & 24 \\
\hline $\begin{array}{l}\text { Peak systolic annular velocity RV } \\
(\mathrm{cm} / \mathrm{s})\end{array}$ & $2.8(0.9)$ & 28 \\
\hline Displacement LV (mm) & $2.1(0.8)$ & 24 \\
\hline Displacement RV (mm) & $3.4(1.3)$ & 28 \\
\hline
\end{tabular}

[Results of all measurements]

No correlations were found between systolic myocardial function, BP and SVC flow ( $p>0.3)$.

Conclusion: The lack of significant correlations between TDI measurements of myocardial function, BP and SVC flow indicate that these parameters are influenced by other factors. Larger studies are need to elucidate these factors. 